\title{
How Fair is Fairness-aware Representative Ranking?
}

\author{
Akrati Saxena \\ Department of Mathematics and \\ Computer Science \\ Eindhoven University of Technology \\ The Netherlands \\ a.saxena@tue.nl
}

\author{
George Fletcher \\ Department of Mathematics and \\ Computer Science \\ Eindhoven University of Technology \\ The Netherlands \\ g.h.l.fletcher@tue.nl
}

\author{
Mykola Pechenizkiy \\ Department of Mathematics and \\ Computer Science \\ Eindhoven University of Technology \\ The Netherlands \\ m.pechenizkiy@tue.nl
}

\begin{abstract}
It has been observed in several works that the ranking of candidates based on their score can be biased for candidates belonging to the minority community. In recent works, the fairness-aware representative ranking was proposed for computing fairness-aware re-ranking of results. The proposed algorithm achieves the desired distribution of top-ranked results with respect to one or more protected attributes. In this work, we highlight the bias in fairnessaware representative ranking for an individual and for a group if the group is sub-active on the platform. We define individual unfairness and group unfairness from two different perspectives. We further propose methods to generate ideal individual and group fair representative ranking if the universal representation ratio is known. The paper is concluded with open challenges and further directions.
\end{abstract}

\section{CCS CONCEPTS}

- Information systems $\rightarrow$ Learning to rank; Information retrieval diversity.

\section{KEYWORDS}

Fairness, Representative Ranking, Bias

\section{ACM Reference Format:}

Akrati Saxena, George Fletcher, and Mykola Pechenizkiy. 2021. How Fair is Fairness-aware Representative Ranking?. In Companion Proceedings of the Web Conference 2021 (WWW'21 Companion), April 19-23, 2021, Ljubljana, Slovenia. ACM, New York, NY, USA, 5 pages. https://doi.org/10.1145/3442442. 3453458

\section{INTRODUCTION}

The ranking problem is encountered in many different applications, including ordering job candidates, providing results for search queries, providing suggestions and recommendations $[4,6,16]$. There are several works that have highlighted the bias in placing the elements in top- $k$ returned results $[12,19,20]$. It is important to understand the limitations of these methods and update the models to achieve fair outcomes for sensitive scenarios as they have been applied to everyday applications [7-9]. There are several works on

This paper is published under the Creative Commons Attribution 4.0 International (CC-BY 4.0) license. Authors reserve their rights to disseminate the work on their personal and corporate Web sites with the appropriate attribution.

WWW'21 Companion, April 19-23, 2021, Ljubljana, Slovenia

(c) 2021 IW3C2 (International World Wide Web Conference Committee), published under Creative Commons CC-BY 4.0 License.

ACM ISBN 978-1-4503-8313-4/21/04

https://doi.org/10.1145/3442442.3453458 proposing fairness-aware ranking methods, including $[1,2,4,5,18-$ 20]. The recent works have investigated both notions of fairness, (i) individual fairness [3, 8, 11], and (ii) group fairness [13-15].

Geyik et al. [10] proposed fairness-aware representative ranking (FRR) maintains the ratio of candidates for each category in top$\mathrm{k}$ elements based on their ratio in the eligible candidates so that each group will get a fair number of resources. They verified the proposed method by applying FRR on LinkedIn Talent Search and observed three-times improvement in the fairness without affecting the quality of the results. However, there is one big concern with the proposed method is what representation ratio for each group of candidates based on protected attributes should be maintained? We show that the deserving candidates of a group miss out on the opportunities by getting ranked lower in the returned list if the universal representation ratio of the candidates is not known.

Example. Let's assume that for a search query $r$, the set of eligible candidates in Universe is represented by $U$ and the set of eligible candidates on a platform $\mathbb{L}$ is represented by $L$. For a protected attribute $A$ having two values the candidates are divided into two groups $G_{1}$ and $G_{2}$. The elements belonging to group $G_{1}$ are $\left\{b_{1}, b_{2}, b_{3}, b_{4}, b_{5}, b_{6}, b_{7}, b_{8}, b_{9}, b_{10}\right\}$, and the ranking of candidates is $b_{1}>b_{2}>b_{3}>b_{4}>b_{5}>\ldots>b_{10}$, therefore candidate $b_{1}$ has the highest ranking 1 . The candidates belonging to group $G_{2}$ are $\left\{g_{1}, g_{2}, g_{3}, g_{4}, g_{5}\right\}$ and their ranking is $g_{1}>g_{2}>g_{3}>$ $g_{4}>g_{5}$. Now, we assume that all candidates of group $G_{1}$ joins the platform $\mathbb{L}$, and group $G_{2}$ is sub-active (or less active) on platform $\mathbb{L}$, therefore, only two candidates $\left(g_{1}, g_{2}\right)$ join the platform $\mathbb{L}$ as shown in Figure 1. Both set $U$ and $L$ will apply fair representative ranking by maintaining the ratio of candidates for each protected group when top- $k$ candidates are requested; The fair representative ranking is explained in detail in Section 2.1. In this example, set $U$ will maintain the ratio $\left(G_{1}: G_{2}\right)=(10: 5)$ and set $L$ will maintain the ratio $\left(G_{1}: G_{2}\right)=(10: 2)$.

If an employer requests top- 6 candidates for search query $r$ : - $U$ will return: $\left(b_{1}>g_{1}>b_{2}>b_{3}>g_{2}>b_{4}\right)$.

- $L$ will return: $\left(b_{1}>b_{2}>b_{3}>g_{1}>b_{4}>b_{5}\right)$.

You can observe that even though $g_{2}$ deserves the opportunity and is active on platform $\mathbb{L}$, the candidate has not been selected in top- 6 when the FRR was applied on the platform $\mathbb{L}$. The main reason for this unfairness is that the representative ranking on platform $\mathbb{L}(L R R)$ maintained the candidates' ratio for protected attributes based on $L$ and did not consider the universal representation ratio. Therefore, the eligible candidates miss the opportunity if other members belonging to that group either do not join or are subactive on the platform.

This paper presents how the existing approach of fairness representative ranking is inadequate to generate a fair ranking given 


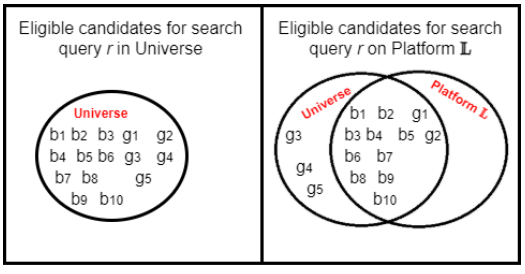

Figure 1: Example for displaying the unfairness in representative ranking for a sub-active group.

the protected attributes. We also present the definitions of unfairness that should be considered while proposing a fairness-aware ranking method. We discuss how the system should be extended to mitigate bias in ranking problems if the complete information is unknown. We discuss open challenges associated with the problem while applying fair representative ranking and further directions that might be considered for solving these challenges.

\section{PREREQUISITE}

Fair Representative Ranking. The FRR method works as follows. (i) Partition the set of qualified candidates into different groups based on protected attribute values. For example, in the case of gender, the candidates will be divided into two groups, males and females. (ii) Rank the candidates in each group according to their scores. (iii) To generate representative ranking, merge these groups in such a way that the proportion of each group in the ranked list for every index of the list is similar to their corresponding proportions in the set of qualified candidates.

Terminologies. The protected attribute are represented as $A=$ $\left\{a_{1}, a_{2}, a_{3}, \ldots . a_{l}\right\}$. For a candidate, its protected attribute is denoted by $A\left(c_{i}\right)$. For the given protected attributes $A$, the qualified candidates can be divided into $n$ groups $\left(G_{1}, G_{2}, \ldots G_{n}\right)$. The fraction of eligible candidates for each protected value in $U$ is $\left\{p_{a_{1}}, p_{a_{2}}, \ldots, p_{a_{l}}\right\}$. $\tau_{U, r, A}$ represents the representative ranking of candidates from set $U$ for the search query $r$, based on protected attribute $A . \tau_{U, r, A}^{k}$ denotes top $k$ elements in the ranking. $\tau_{U, r, A}$ and $\tau_{L, r, A}$ are also referred as URR and LRR, respectively.

\section{FAIRNESS DEFINITIONS}

\subsection{Benefited Candidate}

Definition 1. For the given set $L$, skill set $r$, protected attribute $A$, and top- $k$ selected candidates, a candidate $c_{i}$ is benefited candidate if $c_{i} \in \tau_{L, r, A}^{k}$.

\subsection{Individual Unfairness}

Definition 2. For the given set $U$ and $L$, search query $r$, and protected attribute $A$, if top- $k$ candidates are selected from $L$, the individual unfairness for a candidate $c_{i}$ is defined as, $\operatorname{IUF}\left(c_{i}\right)=1$, if $\left(c_{i} \notin \tau_{L, r, A}^{k}\right.$ and $\left.c_{i} \in \tau_{U, r, A}^{k}\right)$, and 0 , otherwise.

\subsection{Favored Candidate}

Definition 3. For the given set $U$ and $L$, search query $r$, and protected attribute $A$, if top- $k$ candidates are selected from $L$, a candidate $c_{i}$ is favored candidate if, $c_{i} \in \tau_{L, r, A}^{k}$ and $c_{i} \notin \tau_{U, r, A^{*}}^{k}$.

\subsection{Group Unfairness}

Definition 4. In top- $k$ selected candidates, group $G_{i}$ has been treated unfairly by platform $\mathbb{L}$, if $\mid\left\{c_{i} \mid c_{i} \in G_{i}\right.$ and $\left.c_{i} \in \tau_{L, r, A}^{k}\right\} \mid<$ $\mid\left\{c_{i} \mid c_{i} \in G_{i}\right.$ and $\left.c_{i} \in \tau_{U, r, A}^{k}\right\} \mid$.

\subsection{Favored Group}

Definition 5. In top- $k$ selected candidates, group $G_{i}$ is favored by platform $\mathbb{L}$, if $\mid\left\{c_{i} \mid c_{i} \in G_{i}\right.$ and $\left.c_{i} \in \tau_{L, r, A}^{k}\right\}|>|\left\{c_{i} \mid c_{i} \in G_{i}\right.$ and $c_{i} \in$ $\left.\tau_{U, r, A}^{k}\right\} \mid$.

\subsection{Relation of different Definitions}

\subsubsection{Individual Unfairness v.s. Favored Candidate.}

Proposition 1. In top- $k$ selected candidates, if a candidate $c_{i}$ from group $G_{i}$ is treated unfairly as defined in Definition 2, then a candidate from any other group will be favored as defined in Definition 3.

Proof. We prove it using proof of contradiction. Let's assume there are two groups and a candidate $c_{i}$ from group $G_{1}$ is treated unfairly and no candidate of group $G_{2}$ is favored. In top-k elements, URR will return $\operatorname{round}\left(p_{a_{1}} * k\right)$ candidates from group $G_{1}$ and round $\left(p_{a_{2}} * k\right)$ candidates from group $G_{2}$, where $\operatorname{round}\left(p_{a_{1}} *\right.$ $k)+\operatorname{round}\left(p_{a_{2}} * k\right)=k$.

Now, if the candidate $c_{i}$ Group $G_{1}$ is treated unfairly then $c_{i} \notin$ $\tau_{L, r, A}^{k}$ and $\tau_{L, r, A}^{k}\left(c_{i}\right)>k$, so the place of $c_{i}$ candidate in top- $k$ candidates will be vacant. Therefore, the platform $\mathbb{L}$ will return $\operatorname{round}\left(p_{a_{1}} * k\right)-1$ candidates from group $G_{1}$ and $\operatorname{round}\left(p_{a_{2}} * k\right)$ candidates from group $G_{2}$ as no candidate from group $G_{2}$ is favored. Now, the total number of returned candidates by platform $\mathbb{L}$ is $\operatorname{round}\left(p_{a_{1}} * k\right)-1+\operatorname{round}\left(p_{a_{2}} * k\right)=k-1$. That is a contradiction as top- $k$ elements were selected by $L$. Therefore, by the proof of contradiction, an element from group $G_{2}$ will be favored.

3.6.2 Favored Candidate v.s. Individual Unfairness. Intuitively it seems, If a candidate from group $G_{i}$ is favored, then a candidate from any other group $G_{j}$ will be treated individually unfairly as the total number of selected candidates are $k$. However, this is not always true.

Proof. We prove it using the proof of construction. Let's assume, in the example (Figure 1), only $g_{2}$ and $g_{4}$ joins the platform $\mathbb{L}$. Now $\left(G_{1}: G_{2}=10: 2\right)$, so, the new representative ranking in set $L$ is: $b_{1}>b_{2}>b_{3}>g_{2}>b_{4}>b_{5}>b_{6}>b_{7}>b_{8}>g_{4}>b_{9}>b_{10}$. If top-6 candidates are selected,

- U will return: $b_{1}>g_{1}>b_{2}>b_{3}>g_{2}>b_{4}$

- L will return: $b_{1}>b_{2}>b_{3}>g_{2}>b_{4}>b_{5}$

As you can see that the candidate $b_{5}$ is favored but no candidate of group $G_{2}$ is treated unfairly. Hence proved.

\subsubsection{Individual Unfairness v.s. Group Unfairness.}

LEMma 1. If there is individual unfairness (as defined in Definition 2) for a candidate $c_{i}$ and $c_{i} \in G_{i}$, it implies the group unfairness (as defined in Definition 4) for group $G_{i}$.

\subsubsection{Favored Group v.s. Group Unfairness.}

LemMA 2. If there exists a group $G_{i}$ that is favored group, then it implies that there will also exist a group $G_{j}$ that suffers through group unfairness. 


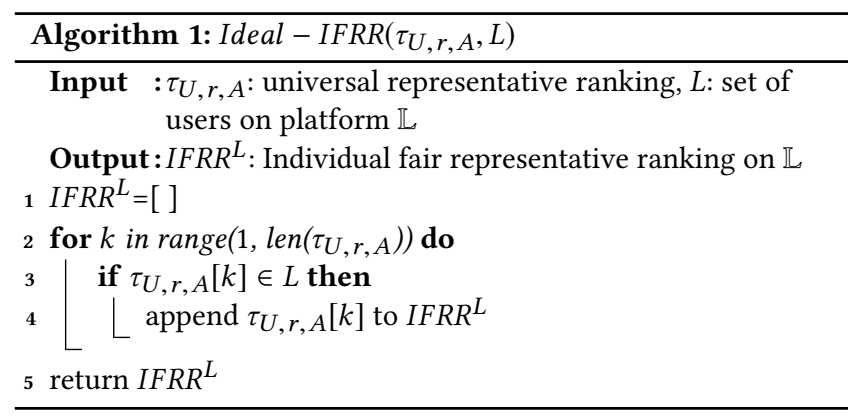

3.6.5 Individual Fairness v.s. Group Fairness. This is important to understand how individual and group fairness are correlated so that the appropriate solutions can be designed.

Lemma 3. The individual fairness-aware solution does not imply group fairness.

Proof. Let's assume, in top- $k$ candidates, the number of chosen candidates by URR from groups $\left(G_{1}, \cdots, G_{i}, \cdots, G_{2}\right)$ are $\left(l_{G_{1}}^{U}, \cdots, l_{G_{i}}^{U}, \cdots, l_{G_{2}}^{U}\right)$, respectively, and the candidates in set $H$ $\left(H=\left\{c_{i} \mid c_{i} \in G_{i}\right.\right.$ and $\left.\left.c_{i} \in \tau_{U, r, A}^{k}\right\}\right)$ does not join the platform $\mathbb{L}$.

In this case, even if no candidate of $G_{i}$ is chosen by an algorithm in top- $k$ candidates, the algorithm is still providing an individualfair solution as there is no individual unfairness as per Definition 2 ; however, it is not a group-fair solution, as $l_{G_{i}}^{U}$ candidates from group $G_{i}$ should have been chosen in top- $k$ for adhering the group fairness as per the Definition 4. Hence proved.

Lemma 4. Group fairness-aware solution will imply individual aware-fairness.

Refer to [17] for the proof.

\section{FAIR REPRESENTATIVE RANKING}

In this section, we discuss what is an ideal solution for ideal fair representative ranking on a platform $\mathbb{L}$ if some candidates do not join it.

\subsection{Individual Fair Representative Ranking}

A ranking is fair for an individual $c_{i}$ if $c_{i} \in \tau_{U, r, A}^{k}$ then $c_{i} \in \tau_{L, r, A}^{k}$ IFRR for platform $\mathbb{L}$ can be generated by projecting URR on the candidates of set $L$. It is denoted by $I F R R^{L}$, and the algorithm is given in Algo 1. In the algorithm, range $(1, n)$ function iterates from 1 to $n$ and $\operatorname{len}\left(\tau_{U, r, A}\right)$ function returns the length of the given list. In steps 2 to 4 , we iterate over each candidate $c_{i}$ of the URR, and if the candidate belongs to set $L\left(c_{i} \in L\right)$, it is added to IFRR ${ }^{L}$.

\subsection{Group Fair Representative Ranking}

A ranking solution is group fair representative ranking (GFRR) if no group $G_{i}$ satisfies the condition of group unfairness as defined in Definition 4. For each index $k$, an ideal-GFRR solution will maintain the group fairness for each group $G_{i}$ until all the elements of that group are exhausted. Therefore, a ranking is fair for a group $G_{i}$ having protected value $a_{i}$ in $A$, if $\mid c_{i}$ s.t. $c_{i} \in \tau_{L, r, A}^{k}$ and $A\left(c_{i}\right)=a_{i} \mid=$ $\min \left(\mid c_{i}\right.$ s.t. $c_{i} \in \tau_{U, r, A}^{k}$ and $A\left(c_{i}\right)=a_{i}|,| c_{i}$ s.t. $c_{i} \in L$ and $\left.A\left(c_{i}\right)=a_{i} \mid\right)$.

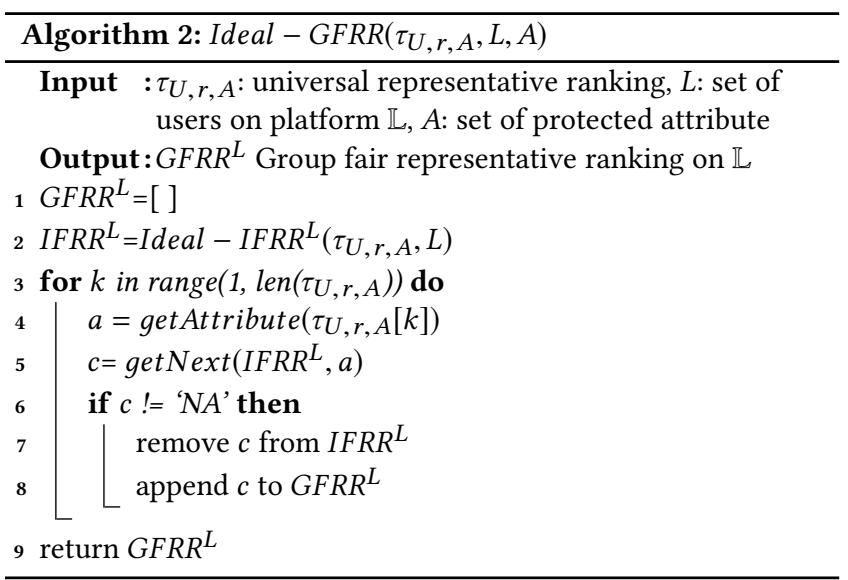

It is denoted by $G F R R^{L}$, and the method is explained in Algorithm 2. For each index $k$ of URR, if the candidate $\tau_{U, r, A}[k]$ is in set $L$, it is added to $G F R R^{L}$; otherwise, the next eligible candidate of this protected group is added to $G F R R^{L}$. The getAttribute(c) function returns the protected attribute of candidate $c, \operatorname{get} \operatorname{Next}(R, a)$ function returns the first element of ranked list $R$ having protected attribute $a$, if there is no remaining element having this attribute, it returns 'NA'.

\section{PROBLEM STATEMENT}

If the URR is known, then IFRR ${ }^{L}$ and $G F R R^{L}$ can be generated using Algorithm 1 and 2; however, it is unknown in most real-world applications. For example, for a given skill set $s$, how many males and females candidates are eligible (the universal representation ratio) might not be known to any given platform $\mathbb{L}$.

Problem Statement. Given set $L$ containing eligible candidates for a search query $r$, protected attribute set $A$, and the number of selected candidates $k$, propose methods to generate optimal IFRR and GFRR.

The proposed solutions should maintain IFRR or GFRR based on the representation ratio of different classes of protected attributes. Therefore, the solutions are categorized into two following classes: (i) If universal representation ratio is known: In this work, we will propose solutions and possible approaches to generate optimal IFRR and GFRR solutions, and (ii) If universal representation ratio is unknown: Here, we first design methods to estimate the universal representation ratio and then the solutions proposed for the first category can be applied. These solutions are not covered here.

\section{THE PROPOSED SOLUTIONS}

In this section, first, we discuss a method to generate an optimal solution for GFRR. The solution to generate optimal IFRR is not straight forward as IFRR ranking is highly dependent on the kind of missing data; we will discuss these complexities and possible IFRR solution in the next subsection.

\subsection{Generate GFRR}

The method to generate GFRR will maintain the given ratio of candidates based on protected attribute classes for each index $k$. 


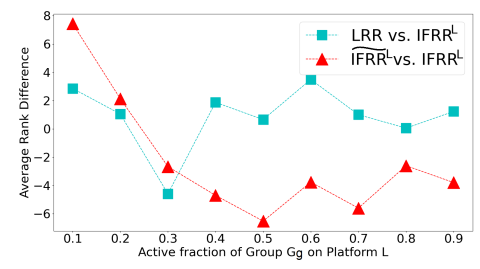

Figure 2: Average Rank Difference versus active fraction of group $G_{g}$ for group $G_{g}$.

The method is inspired from [10], and the generated GFRR ranking is represented by $\widehat{G F R R^{L}}$. The generated solution will be an Ideal-GFRR; as both $\widehat{G F R R^{L}}$ and $G F R R^{L}$ rankings maintain the representation ratio for each index $k$.

\subsection{Generate IFRR}

The solutions to generate IFRR need more information, even if the universal representation ratio is known. We will explain it with the help of an example. Let's assume, in the universal set $U$ there are two groups $\left(G_{1}, G_{2}\right)$ and for search query $r$, in group $G_{1}$, there are four eligible candidates $\left(b_{1}, b_{2}, b_{3}, b_{4}\right)$ and in group $G_{2}$, there are four eligible candidates $\left(g_{1}, g_{2}, g_{3}, g_{4}\right)$. We assume that in each group the rank of $c_{i}>c_{j}$ if $i<j$. So, IFRR on set $U$ is $\left(g_{1}, b_{1}, g_{2}, b_{2}, g_{3}, b_{3}, g_{4}, b_{4}\right)$ if group $G_{2}$ is prioritized. Let's assume that four candidates from group $G_{1}$ and two candidates from group $G_{2}$ joins the platform $\mathbb{L}$, therefore $L=\left\{b_{1}, b_{2}, b_{3}, b_{4}, g_{1}^{\prime}, g_{2}^{\prime}\right\}$. Now, the question is: if the universal representation ratio is known as (0.5:0.5), how to generate an individual fair representative ranking. It is not straight forward as we do not know which two out of four members of group $G_{2}$ have joined $L$. Let's further understand with case scenarios. In case 1 : If $g_{1}^{\prime}$ is $g_{1}$ and $g_{2}^{\prime}$ is $g_{2}$, then the Ideal-IFRR will be $\left(g_{1}, b_{1}, g_{2}, b_{2}, b_{3}, b_{4}\right)$. In case 2 : If $g_{1}^{\prime}$ is $g_{1}$ and $g_{2}^{\prime}$ is $g_{4}$, then the Ideal-IFRR will be $\left(g_{1}, b_{1}, b_{2}, b_{3}, g_{4}, b_{4}\right)$. Therefore, an IFRR solution also require the knowledge of missing data or the knowledge of what kind of candidates have joined $\mathbb{L}$.

The information of what kind of candidates have joined the platform $\mathbb{L}$ can either be gathered by surveys or by using statistical methods. The surveys can help in knowing what is the probability of a candidate joining $L$ and what is its correlation with the candidate's characteristics. For example, if we know that the higher-ranked people are more likely to join a platform $\mathbb{L}$, then one estimated IFRR solution for the above-discussed example is: $\left(g_{1}^{\prime}, b_{1}, g_{2}^{\prime}, b_{2}, b_{3}, b_{4}\right)$ as shown in case 1 . In another approach, the statistical methods can be used to estimate the actual distribution using the partial available information. In this work, we provide a solution for the case when the candidates from a given group $G_{i}$ are missing uniformly at random. In the proposed solution, for each ranking place reserved for a sub-active group, its candidate will be placed with the probability equal to the fraction of active people of this group. The method is represented by $\widehat{I F R R^{L}}$.

\section{SIMULATION RESULTS}

Datasets. The experimental results are shown on synthetic datasets so that the impact of different activeness can be better studied and

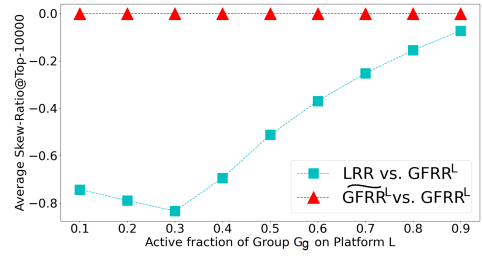

Figure 3: Average Skew ratio versus active fraction of group $G_{g}$ for top-10000 ranking for group $G_{g}$.

various values of protected attributes can be considered. The data has a protected attribute having 3 values $A=\{g, b, u\}$ and so the candidates are categorized into three groups $G_{g}, G_{b}, G_{u}$. A realworld example of this situation is gender classes having 'male', 'female', and 'unknown' values. For a search query $r$, in each group, there are 10000 eligible candidates, and their scores are assigned uniformly at random from $(0,1]$. We assume that group $G_{g}$ is less active on the platform $\mathbb{L}$, and the ratio of active members ranges from 0.1 to 0.9 ; and all candidates of $G_{b}$ and $G_{u}$ join $\mathbb{L}$.

Metrics. Rank Difference is used to capture the individual unfairness for ranking $R_{1}$ v.s. ranking $R_{2}$. It is computed as,

RankDiffer $\left(c_{i}\right)=R_{1}\left(c_{i}\right)-R_{2}\left(c_{i}\right)$.

Skew ratio measures group unfairness and denotes the extent to which the top-k elements for a given search query and for a given sub-group differ from the desired proportion. It is computed as,

$\operatorname{Skew}_{R_{1}, R_{2}}^{a_{i}}=\log _{e}\left(\frac{\mid\left\{c_{i} \text { s.t. } c_{i} \in R_{1}^{k} \text { and } A\left(c_{i}\right)=a_{i}\right\} \mid}{\mid\left\{c_{i} \text { s.t. } c_{i} \in R_{2}^{k} \text { and } A\left(c_{i}\right)=a_{i}\right\} \mid}\right)$.

Discussion. Figure 2 shows the rank-difference of LRR and the proposed IFRR with Ideal-individual fairness representative ranking. The results show that the performance of LRR is poor for the activeness ratio from 0.4 if the candidates join u.a.r. However, detailed solutions will be proposed further. Figure 3 shows the skewness of LRR versus ideal-group fairness representative ranking, and the proposed method performs as good as the ideal method as both maintain the representation ratio.

\section{CONCLUSION}

In this paper, we discussed how a fairness-aware ranking method might be unfair due to the unavailability of universal information. We proposed individual and group fairness definitions that should be considered while verifying the fairness of the proposed representative ranking if some groups are less active. We further discussed the correlation and dependencies of the proposed definitions. We proposed methods to generate IFRR and GFRR if the universal representation ratio is known. The experiments show preliminary results for unfairness increases in LRR as the group becomes less active and the improvements achieved by proposed methods.

The main challenge in real-life applications is to identify the universal representation ratio based on the given protected attribute. In this work, we highlighted two approaches to solve this (i) perform a survey to understand the existing distribution, (ii) apply statistical methods to estimate the exact distribution based on the available score-ranking information of the candidates belonging to different protected attributes based groups. These solutions will be discussed in future work. 


\section{REFERENCES}

[1] Abolfazl Asudeh, HV Jagadish, Julia Stoyanovich, and Gautam Das. 2019. Designing fair ranking schemes. In Proceedings of the 2019 International Conference on Management of Data. 1259-1276.

[2] Asia J Biega, Krishna P Gummadi, and Gerhard Weikum. 2018. Equity of attention: Amortizing individual fairness in rankings. In The 41st international acm sigir conference on research \& development in information retrieval. 405-414.

[3] Reuben Binns. 2020. On the apparent conflict between individual and group fairness. In Proceedings of the 2020 Conference on Fairness, Accountability, and Transparency. 514-524.

[4] Carlos Castillo. 2019. Fairness and transparency in ranking. In ACM SIGIR Forum, Vol. 52. ACM New York, NY, USA, 64-71.

[5] L Elisa Celis, Damian Straszak, and Nisheeth K Vishnoi. 2017. Ranking with fairness constraints. arXiv preprint arXiv:1704.06840 (2017).

[6] Lee Cohen, Zachary C Lipton, and Yishay Mansour. 2019. Efficient candidate screening under multiple tests and implications for fairness. arXiv preprint arXiv:1905.11361 (2019)

[7] Sam Corbett-Davies, Emma Pierson, Avi Feller, Sharad Goel, and Aziz Huq. 2017 Algorithmic decision making and the cost of fairness. In Proceedings of the $23 \mathrm{rd}$ acm sigkdd international conference on knowledge discovery and data mining. 797-806.

[8] Cynthia Dwork, Moritz Hardt, Toniann Pitassi, Omer Reingold, and Richard Zemel. 2012. Fairness through awareness. In Proceedings of the 3rd innovations in theoretical computer science conference. 214-226.

[9] Sorelle A Friedler, Carlos Scheidegger, and Suresh Venkatasubramanian. 2016. On the (im) possibility of fairness. arXiv preprint arXiv:1609.07236 (2016).

[10] Sahin Cem Geyik, Stuart Ambler, and Krishnaram Kenthapadi. 2019. Fairnessaware ranking in search \& recommendation systems with application to LinkedIn talent search. In Proceedings of the 25th ACM SIGKDD International Conference on Knowledge Discovery \& Data Mining. 2221-2231.

[11] Christina Ilvento. 2019. Metric learning for individual fairness. arXiv preprint arXiv:1906.00250 (2019).
[12] Matthew Kay, Cynthia Matuszek, and Sean A Munson. 2015. Unequal representation and gender stereotypes in image search results for occupations. In Proceedings of the 33rd Annual ACM Conference on Human Factors in Computing Systems. 3819-3828.

[13] Pranay K Lohia, Karthikeyan Natesan Ramamurthy, Manish Bhide, Diptikalyan Saha, Kush R Varshney, and Ruchir Puri. 2019. Bias mitigation post-processing for individual and group fairness. In Icassp 2019-2019 ieee international conference on acoustics, speech and signal processing (icassp). IEEE, 2847-2851.

[14] Dino Pedreschi, Salvatore Ruggieri, and Franco Turini. 2009. Measuring discrimination in socially-sensitive decision records. In Proceedings of the 2009 SIAM international conference on data mining. SIAM, 581-592.

[15] Dino Pedreshi, Salvatore Ruggieri, and Franco Turini. 2008. Discrimination-aware data mining. In Proceedings of the 14th ACM SIGKDD international conference on Knowledge discovery and data mining. 560-568.

[16] Javier Sánchez-Monedero, Lina Dencik, and Lilian Edwards. 2020. What does it mean to'solve'the problem of discrimination in hiring? Social, technical and legal perspectives from the UK on automated hiring systems. In Proceedings of the 2020 conference on fairness, accountability, and transparency. 458-468.

[17] Akrati Saxena, George Fletcher, and Mykola Pechenizkiy. 2021. How Fair is Fairness-aware Representative Ranking and Methods for Fair Ranking. arXiv preprint arXiv:2103.01335 (2021).

[18] Ashudeep Singh and Thorsten Joachims. 2018. Fairness of exposure in rankings. In Proceedings of the 24th ACM SIGKDD International Conference on Knowledge Discovery \& Data Mining. 2219-2228.

[19] Ke Yang and Julia Stoyanovich. 2017. Measuring fairness in ranked outputs. In Proceedings of the 29th International Conference on Scientific and Statistical Database Management. 1-6.

[20] Meike Zehlike, Francesco Bonchi, Carlos Castillo, Sara Hajian, Mohamed Megahed, and Ricardo Baeza-Yates. 2017. Fa* ir: A fair top-k ranking algorithm. In Proceedings of the 2017 ACM on Conference on Information and Knowledge Management. $1569-1578$. 